\title{
Stefan Bajohr/Kathrin Rödiger-Bajohr Die Diskriminierung der Juristin in Deutschland bis 1945
}

Im Januar 1973 erschien in der "Deurschen Richterzeirung" (DRiZ) ein kurzer Beitrag zur "Benachreiligung der Juriscin zwischen 1933 und 1945*, der den Eindruck erweckte, als habe es sich hierbei um eine lediglich aus dem Antifeminismus der NSDAP erklärbare Diskriminierung gehandelt. Durch die Herauslösung der Phase 1933-1945 wird dorr die Diskriminierung der Frauen durch die Juristenschaft umgemünzt in eine Diskriminierung der Juristinnen durch den Faschismus (an der die Juristenschaft im übrigen nicht beteiligt zu sein schien). Hierbei bleibr die Tarsache unbeachret, daß der Faschismus mit seinen gegen die Juristinnen gerichceten Maßnahmen den frauenfeindlichen Atritüden der Mehrzahl der männlichen Juristen durchaus entgegenkam. Die Darstellung der Diskriminierung der Juristinnen während der Zeir der faschistischen Herrschaft in Deutschland muß daher in den Zusammenhang der vehementen Ablehnung gestells werden, die die Juristenschaft auch vor 1933 den weiblichen Kollegen entgegenbrachte.

Dabei geht es um zweierlei: Erstens kann am Beispiel der Lage der Juristinnen während der ersten Hälfre dieses Jahrhunderts die erhebliche Zurückserzung der gebildeten Frau im Beruf besonders prägnant herausgearbeiter werden, da sich in der Halrung gegenüber der juristischen Ausbildung von Frauen und gegenüber juriscisch ausgebildeten Frauen nahezu all jene Formen der Diskriminierung bündelten, die einzeln auch andere Gruppen von Akademikerinnen trafen. Dies erklärr sich wohl am ehesten daraus, daß der Beruf der Juristin, anders als beispielsweise der der Lehrerin, Aporhekerin, Veterinär-, Zahn- und Humanmedizinerin, nicht jenem Berärigungsfeld zuzurechnen war (ist), àuf dem, entsprechend der traditionell vorgegebenen Rollenverteilung (die dem weiblichen Geschlecht erzieherische und pflegerische Aufgaben zuweist), die Frauen, trotz allen Mißtrauens und Widerstandes, gerade noch gedulder wurden. Mit der Ausübung eines juristischen Berufes drohten sie jedoch in die von den Männern sorgsam gehüteten warcana imperiij einzudringen und damit einen Einfluß auf politische und gesellschaftliche Vorgänge zu nehmen, der ihnen trocz der 1919 formal gegebenen Gleichberechrigung nicht zugestanden wurde. Über die historische Aufarbeitung der extremen Intransigenz männlich beherrschter Parlamente, Regierungen und Behörden gegenüber den Juristinnen kann aber zweirens das bei der Mehrzahl bundesdeurscher Juristen relativ ungebrochene Verhälınis zur Tradicion der eigenen Berufsgruppe ${ }^{3}$ in Frage

I Anne-Gudrun Meier-Scherling: Dic Benachteiligung der Juriscin zorischen 1933 und 1945, in: DRjZ, 53. Jg., 1975, S. 10-13.

2 Vgl. Stefan Bajohr: Dic Hälfec der Fabrik. Geschichte der Frauenarbeit in Deutschland 1914-1945, Marburg 1979, S. 219 if.

3 Ein ungctruberes Tradicionsbewußtsein aus jungster Zeir finder sich bei Gen März: Zur Lage der Justiz, in: DRiZ, 56. Jg., 1978, S. 101-10), besonders S. 101. Vgl. auch Wilhelm Henke: Wider dic Policisicrung der Justiz, in: DRiZ, s2. Jg., 1974, S. 177. Ganz allgemcin zum Traditionsverständnis vgl.

hur viele: Richard Schmid: Justiz in der Bundesrepublik, Pfullingen 1967, S, 20. 
gestellt werden: Die Berufung auf die "guten Tradirionen « der deurschen Juristenschafr beinhalter eben auch die Bejahung des Antifeminismus, die Befurrwortung der Diskriminierung von Menschen auf Grund ihres Geschlechrs. Dieses Traditionsverszünd’nis zu erschüttern, soll dieser Aufsatz beitragen.

wöge der Tag nicht mehr fern sein, an dem es nicht mehr bloß eine Allegorie sein wird, daß eine Frau die Waage der Gerechtigkeit in den Händen hälc. ${ }^{4} \mathrm{Als}$ Josef $\mathrm{T}$. Goldberger im Juni 1919 diese Hoffnung äußerte, schien es, als härte die bürgerlichliberale Frauenbewegungs in Deutschland den entscheidenden Sieg im Kampl um die jurissischen Ämter, diese letzte Bastion männlicher Alleinherrschaft in den akademischen Berufen, so gut wie errungen.

Nachdem sie sich bis Ende 1908/09 Universitäı für Universität zugänglich gemachs hatten und seirdem in ganz Deutschland als ordentliche Studierende zugelassen worden waren, ${ }^{6}$ schien mit dem Ende des Ersten Weltkriegs und dem Sturz der Monarchie der Augenblick gekommen zu sein, da die Frauen auch zu den juristischen Examina zugelassen würden, deren erstes die Vorbedingung für die Aufnahme in den juristischen Vorbereitungsdienst (das Referendariat) und deren zweites die Voraussetzung für die Ausübung eines juristischen Berufs bildece.? Verstärkt wurde diese Erwarrung noch durch die am 11. August 1919 erfolgte Verabschiedung der republikanischen Reichsverfassung, in deren Art. 109 Abs. I es hieß: "Alle Deurschen sind vor dem Gesetz gleich. Männer und Frauen haben grundsäzzlich dieselben staatsbürgerlichen Rechte und Pflichten." Insbesondere verrraute man auch darauf, daß der Verfassungsauftrag aus Arl. $228^{8}$ sich bald in einer Neufassung derjenigen Vorschriften niederschlagen würde, die den Frauen noch immer die Ausübung eines juristischen Berufs verwehrcen. ${ }^{9}$ Doch es dauerte noch knapp drei Jahre, bis am 11. Juli 1922 durch das "Gesetz über die Zulassung der Frauen zu den Ämtern und Berufen der Rechtspflege ${ }^{10}$ die letzten formalen Hindernisse beiseite geräume wurden. Damit schien ein unumkehrbarer Durchbruch gelungen zu sein, und wohl niemand hätte vermutet, daß schon zwölf Jahre später erneut versucht werden würde, den Fraven die Ausübung juristischer Berufe zu verwehren.

Der Ausschluß der Frauen von den juristischen Berufen stützte sich vor 1922 auf

4 Josef T. Goldberger: Vom Berufe der Frauen unserer Zeit für dic Rechtspflegc, in: Dic Frau, 26. Jg., $1918 / 19$, S. $279-278$. S. 278

s Gegen die Mirwirkung der Frauen an der Rechuspilege waren unrer den bürgerlichen Frauenorganisa. vionen lediglich einige katholische und rechisextrenistische Verbände cingestells. Vgl. Otro Harwig Die Frau in der Rechuspilege. Systemarische Darstchlung der Beteiligung der Frau an der Reclusppllege im In- und Auslande (" Meyersche Gesetzsammlung), Dermold 1922, S. 10.

6 Jena hatle als \{etzic Universitat in Deutschland im Jahre rgoz Fraucn zum Jurastudium zugelassen. Vgl. Erna v. Langsdorff: Dic Berufsaussichen der Juristin in Deutschland, in: Dic Frau, 20. Jg., 1912/13. S. $603-609$, 5. 603 . Zu dem langwierigen Prozeß der Zulasssung von Fraucn zum Universitarsstudium in Deurschland vgl. Gerraude Schnelle: Zur Geschichre des Fraverisrudiums in Deurschland, Berlin r 97 I, und Renalc Schopi: Dic Bedeutung des Frauenstudiums für die Stellung der Frau in der Gesellschad und im Enwerbsleben. Dic Enıwicklung des Fraucnstudiums von der Jahrhundenwende bis heute. Stuingar igs?

$7 \$ 2$ Abs. , GVG vom 27. 1. 1877 (RGBl. 18 77, S. 41-76, S. 31 ).

8 mAlle Seaalsbürger ohne Unterschied sind nach Maßgabe der Geserze und entsprechend ihrer Befähigung und ihren Leistungen zu den öffentlichen Xmtern zuzulassen. Alle Ausnahmebestimmungen gegen weibliche Beamee werden bescivige. Die Grondlagen des Bcamenverhälınisses sind durch Reichsgesetz zu regeln.a (Dic Verfassung des Deurschen Reichs vom 11. Auguss 1919, Art, 128). Das Wore "wendens in Salz a des Art, 128 hae übrigens zu Konrrovirsen gefuhhr. So wurde gegen die Ansichx, es sei damit die solorige tarsachhche Aufhebung aller Ausnahmebestimmungen gegen weibliche Bcamtc gemeint und bezweckt, eingewandt, daß wwerden " lediglich einc Anweisung für dic Zukunit, einen Verfassungsaultrag also, bejohalec (vgl. Bundesarchiv Koblenz (BA) Nachlaß Luders vorl. N. (4 48 ).

9 Vgl. die diesbezügliche Antrage Nr. I 39 (Aktenstück Nr. 249) vom 28. 7. 1920 an die Reichsregicrung, in: Verhandlungen des Reichsiags, I. Wahlperiode 1920 , Bd. 363, Berlin 1924, S $206 \mathrm{f}$.

10 RGBI. 19221 , S. 573 I. 
eine durchaus zweifelhafre Rechrsgrundlage. Die einschlägigen Geserze, die aus den I $870 e$ J Jahren stammien, enchielten nämlich keineswegs ein ausdrückliches Verbor der Ausübung juristischer Berufe durch Frauen. " Dies erklärt sich daraus, daß zu jener Zeit ein explizirer Ausschluß des weiblichen Geschlechrs überflussig erschien, weil die Universitätsausbildung den Frauen noch verschlossen war und sich folglich das Problem einer Zulassung von Jurastudentinnen zur ersten Scaatsprüfung nicht stellre. Als die Frauen mir der Offnung der Universiräten für weibliche Studierende nun auch die juristischen Fächer belegren, verweigerte man ihnen die Ablegung der Staatsexamina mit dem Argument, eine Zulassung der Frau zum Richceramt, zur Rechts- und Staatsanwaltschaft sei mit dem geltenden Recht niche zu vereinbaren." Daß ausschließlich ein Mann zum Richter bzw. Rcchrsanwalt befähigt sei, folgerte man u. a. aus $\S_{4}$ I Zifl. 3 ZPO, wonach der Richter von der Ausübung seines Amtes ausgeschiossen war win Sachen seiner Ehelrau* (nicht: seines Ehegatten). "In derselben Weise interpretierte man auch die Vorschriften der $\$ \$ 22 \mathrm{StPO}, 156 \mathrm{GVG}$ und 6 FGG. ' 4 Schließlich berief man sich auf die Intention des BGB-Gesetzgebers, der bei der Schaffung des BGB als selbstverständlich davon ausgegangen sei, daß ausschließlich Männer zum Richıeramt befähigt seien."

Einige der Fraven, die, obwohl ihnen bis 1922 die Ausübung eines juristischen Berufes versagt war, bereits vor 1914 ein Jurascudium begonnen hatten, hatten dies zum Teil bis zu einem ersten Abschluß geführt. Dieser bestand, da den weiblichen Jurascudenten die Zulassung zur ersten Staatsprüfung in allen deutschen Ländern - mit Ausnahme von Bayern' - verweigert wurde, ausschließlich in der Promotion. $1913 \mathrm{gab}$ es in Deutschland zwölf Frauen, die ihre Jurastudien mit dem Erwerb des Doktortitels abgeschlossen hatten; ${ }^{17}$ allerdings waren sie vorwiegend nicht in dem gelernten Fach, sondera in anderen Berufcn rätig. ${ }^{18}$

Die Zahl der Jurastudentinnen, die im Sommersemester 191347 betragen hatte, ${ }^{19}$

n Vgl. Wolfgang Mittermaicr: Wic studien man Rechuswissenschaf?? Das Sudium der Rechiswissenschaft und scine zweckmäßigc Ennrichnung. Einc Anlcitung lür Studierende, Stuttgant ig11, S. 88; Ema v. Langsdorff, a. a. O., S. 603.

12 Vgl. Adolf Friedländar, M2x Friedländer: Kommentar zur Rechusanwalesordnung vom 1. Juli $1878,2$. Aufl. München/Berlin/Lcip\%ig 1920. S. 1s: Syring: Rezcnsion des Friedlander-Kommentars, in: JW', 48. Jg. 1919, S. $97:$.

13 RGBI. 1877, S. $83-243$, S. 89 ?

14 A. a. O., S. $253-346$, S. 256 ; a. a. O., S. $11-76$, S. 69 I.; RGBI. 1 \$98, S. 189-229, S. 190; vgl, auch Eich: Die Frau in der Rechrsphlege, in: DJZ, 24. Jg., 1919, Sp. 625-629, Sp. 625.

1) Vgl. Adolf Friedländer. Max Friedländer, a. a. O., S. Is; gcgen den Rückgriff aul den vermcintlichen Willen des Geselrgebers in diesem Falle auch Ernst Bahn: Die Frau im Grrichessal, in: Dic Frauenbewegung, 13. Jg., 1907, S. 26-28 u, 36-37, S. 27.

16 In Bayem wunden die Fraucn seit 1903 zur Zwischempröfung und zum ersten juristischen Staatsexamen zugelassen (vgl. Vera Lowitsch: Die Frav als Richeer, Diss. Freiburg i. Br. 1913, S. 62), woraus sich für sie jedoch kein Anrecht auf Ubernahme in den Vorbereitungsdienst herleitete (vgi. a. a. O., 5. 62 f.). Im Jahrc 1910 wurden Frauen dann expressis verbis von der Einstellung in den Vorbereitungsdiensı ausgeschlosscu $\$ 27$ der Königl. VO übor die Vorbedingungen für den hỏheren Jusriz- und Verwaltungsdienst vom 19. 10. 1910, in: Gesctz- und Verordnungsblake lür das Königrcich Baycrn, 1910. S. 1005). Nur cince Ausnahme von diescr Bestimmung ist bekannt (vgl. Wollgang Mirtcrmaier, a. a. O., S. 88).

17 Vgl. Hilde Oppenheimer, Hilde Radomski: Die Probleme der Fraucnarbcil in der Ubergangswirschafr, Mannheim/Berlin/Leipzig 1918, S. 157.

18 Einc dieser $x$ wölf promovierien Jurisunnen war Agnes Marens aus Einbeck, Leiterin der Auskunteste]. le lür Frauenberufe des Verbandes der Fraucnvercine für das Herzogrum Braunschweig (vgl. Stadiarchiv Braunschwsig D IV 5180 und G XI 12 Nr. 1; vgl, auch Nachtrag zum Braunschweigischen Adreßbuch für das Jahr 1913, Braunschwcig :91\}, S. j0). Die anderen waren rätig als wissenschafliche Hilfsarbeitcrin bei der Gewerbeinspektion (1), als wissenschafaliche Assistentin einer stadrischen Rechtsauskunftsstclle (1), als Leirerinnen privater Rechtsschutzstcillen für Frauen (1) als Gescbäftsiührerin einer Jugendfürsargezentrale ( $\mathrm{I}$ ), als Joumalistinnen (2), als lnhaberin cines Rechusauskunfusbüros (1) und als Volontarinnen bei Rechesanwäleen (2) (vgl. Erna v. Langsdorff, 2. 2. O., S. 605).

I9 Vgl. DJZ, 26. Jg.. 1921, Sp. $74^{8}$. 
srieg im Sommersemester 1914 auf 57 , das waren ecwa $1,4 \%$ aller Frauen deutscher Staatsangehörigkeit, die an deutschen Hochschulen und Universitäcen studierten. ${ }^{20}$ Diesen Studentinnen gewährte man nnur eine halbe Ausbildung, die theoretische auf der Universizät, (schloß) . . sie dagegen von der vollen praktischen Vorbildung ... aus. " Während des Ersten Weltkriegs änderte sich an der Praxis der Justizbehörden gegenüber den weiblichen Studierenden niches wesentliches. Lediglich in Bayern wurde einer Juristin, die das erste Staatsexamen abgelegt hatte, infolge Richrermangels eine Hilfsarbeiterinstelle für Vormundschaftssachen angeboren. ${ }^{22}$

Als nach Kriegsende die Forderung nach Zulassung der Frauen zu den juristischen Berufen vehementer vertreten wurde, meldeten sich zahlreiche Stimmen aus juristischen Kreisen, die gegen jegliche Bereiligung der Fraven an der Rechtspflege vorierten. "Alle sollen Kamellene von der Frau im Hause und der sirclichen Würde, die sie fernhält von der Roheit des Kriminalsaales . . . (mußren) herhalten «, "3 um die Ablehnung weiblicher Richter, Rechts- und Sraarsanwälte zu begründen. Darin, daß die Frau "zu schade für den Anwalıs- und Richterberuf $u^{24}$ sei, war sich die Mehrheit der Juristen vollkommen einig. Auch die Ansicht, daß die Frauen auf Grund ihrer wseelische(n) Eigenart, nach welcher sie in weitestgehendem Maße Gefühiseinflüssen unterworfenu seien, "welche ihre sachliche Auffassung beeinträchtigenm,"s wurde von der absoluten Mehrheir männlicher Juristen geteilt. Dazu kam eine offenkundig riefsitzende Angst vor dem nEinfluß gewisser Vorkommnisse im Frauenleben ${ }^{26}{ }^{26}$ der sich auch ndurch die beste Berufsausbildung... nicht so beseitigen (lasse), daß die berufsrichterliche Objektivitäı mir Sicherheit gewährleistec würde «. ${ }^{27}$ Immerhin werde die Frau durch Menstruation, Schwangerschaft und Klimakterium in ihrem Denken und Fühlen so sehr beeinflußt, daß sie häufig "in einen geradezu anormalen Zuscand gerace.$^{28}$ Zwar bescehe die sMöglichkeir der Ausnahme, daß bei einigen wenigen Frauen, welche konstitutionell einen starken männlichen Einschlag haben, die weibliche Mentalität nicht wirksam « werde - doch wer könne mit Gewißheit feststellen, bei welcher Frau dies der Falł sei?29 Weit zurückgewiesen wurde die Ausübung juristischer Berule durch Frauen auch mit

20 Käthe Gaebel: Die Berufslage der Akademikerinnen, in: Die Fruu, 34. Ig, 1926/17, S. $118-1244$. $278-282,5.219 ;$ die Zahi der Jurastudentinnen in Deutschland betrag im WS 1917/18 74, im SS 1919 450 und im SS 1910 sos (vgl. DJZ, 23. Jg., 1918, Sp. 4j0: DJZ, 26. Jg., 1921, Sp. 748). Im W/ 1920/21 wurden an deutschen Universiräten 326 Jurastudenúnnen gezahlt (vgl, Elisaberh Aitmann-Gouhcincr: Dic Bcrulsaussichten der deuschen Akademikerinnen, Halle/Saale 1921, S. 5).

21 Margarere Berent: Dic Zulassung der Frauen zu den juristischen Berulen, in: Dis Frau, 27. Jg., 1919/10, S. 332-334, S. 332.

22 Vgi. Hilde Oppoenhcimer. Hilde Radornski, a. 2. O., S. 208; Erna v. Langsdorff, a. a. O., S. 604, spriche von einer Juristin im Vorbereitungsdienst.

2) Robert Theilhaber: Welbliche Richrer, in: Die Frau im Sraat, 2. Jg. 1920, S. 7-8, S. 7.

24 RA Dr. Kraemer, Berlin, während der Verhandlungen der 14. Vertecerversammlung des Deutschen Anwalusvereins, 28./29. 1, 1912 in Braunschweig, in: JW, \{1. Jg., 1921, S. 1233.

2s Vorrag des Berichterstauers LGDis. Dr. Scadelmann aus Potsdam zum Thema nZulassung der Fraus zum Richteramex am 4. Richterag zu Leipzig (Mai 1911), in: DRiZ, 18. Jg., 1911. Sp. 199; àhnlich Bitcinger: Fraven als Richter?, in: Leigziger Zeitschrift für Deutsches Recht, 13. Jg., 1919, Sp. 676-678, Sp. 677: "Unparteilich zu sein, beruht vor allem auf nazürlicher Anlage... Hier muß nun mit allem Nachdruck ausgesprachen werden, daß die Fruu kratr des natkrgegebenen Unierschieds von den Wesenseigenschaften des Mannes laut allgemeincr Erfahrung dicse Anlage in so viel geringerem Grade besitzt, daß sie zum Richteramt niche geeignet erscheint, *

26 RA Dr. Ebertsheim, Mitberichterstaver während der Verhandlungen der 14. Vertreterversammlung des Deuschen Anwaltsvercins, in: JW, 51 . Jg., 1922, S. 1250.

27 LGDir. De. Suadelmasm, 1. 4. O., Sp. 202.

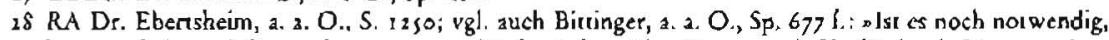
hervorzuheben, $d a B \ldots$ das ganze psychische Leben (der Frauen - d. Verf.) durch Menseruarion, Gravidieär, Klimakterium in ciner Weise beeinflußt wird, dic gerade der richterlichen Tärigkeit besonders aburäglicb scin müßıç."

29 LGDir. Dr. Stadelmann, a. a. O. Sp. 201. 
Hinrveis auf ihre mögliche Beteiligung an Sirtlichkeitsprozessen, da diese eine

unzumurbare Verletzung des den Frauen angeblich naturhaf eigenen „Schamgefühls « mit sich brächten..$^{30} \mathrm{Im}$ übrigen sei für den Juristen ein "autoritatives Aufreten "erforderlich," eine Fähigkeit, über die eine Frau nun einmal nicht verfüge. Ergebnis der Beceiligung der Frauen an der Rechtspflege wäre daher eine "größere Weichheir und Nachgiebigkeitu, die man sich gerade in den ersten Jahren nach der Novemberrevolusion nicht erlauben könne: Schließlich befinde man sich win einer Zeit verrotteter Ordnung «. ${ }^{32}$ Überdies sei es für einen Mann unzumutbar, von einer Frau abgeurteils zu werden, ${ }^{33}$ da eine „Unterstellung des Mannes unter den Willen und Urteilsspruch einer Frau ... der Stellung (widerspreche), welche die Narur dem Manne gegenüber der Frau angewiesen « habe:" "Man solle einmal die Tiere ansehen, die Biene zum Beispiel, bei der sei auch der weiblichen Biene bestimmte Arbeit zugewiesen. So sei es auch bei dem Menschen, es gibt eben bescimmte Berufe und Tätigkeiten, die nicht in den Bereich der Frau gehören, «" ${ }^{35}$ $\mathrm{Da}$ diese Autfassungen durchaus ernst gemeint waren und auch ernst genommen wurden, zeigen die Abstimmungsergebnisse der deutschen Richter- und Rechtsanwaltschaft zur Frage der Zulassung der Frauen zu den juristischen Berulen. Dagegen drangen die wenigen Befürworter einer Beteiligung der Frauen mit ihren Ausführungen nicht durch. Weder Justizrat Dr. Bieber aus Berlin, der den Gleichheitsgrundsatz der Reichsverfassung von 1919 hervorhob, ${ }^{36}$ noch der Geheime Justizrat Dr. Eugen Fuchs, der mic Berufung auf Theodor Mommsen »die Vorenthalcung verfassungsmäßiger Rechte als administrative Prellerei «37 gebrandmarkc hattc: konnten die Ablehnungsfront erschüttern: Sowolyl die Richter ${ }^{8}$ als auch die Rechtsanwälte ${ }^{39}$ lehnten in den Jahren 1920 bis 1922 mit überwältigenden Mehrheiten eine Beteiligung der Frauen an der Rechrspflege ab.

Hinsichtlich der Zulassung von Frauen zum Rechtspraktikum änderte Preußen 1919 seine Haltung dahingehend, daß es Jurastudentinnen preußischer Staatsangehörigkeit in der Regel das erste Examen ablegen ließ, aber weiterhin deren Ernennung zu Referendarinnen ablehnte. $4^{\circ}$ Entsprechendes galı für Bayern. ${ }^{47}$ Weiterhin wurde also den Frauen die Ausübung eines juristischen Berufs unmöglich gemachr. Die

30 Vgl. lür vicle de Niem: Die Fravengerichte nach dem Entwurl zur Abänderung des Gerichevertassungsgeseizes, in: DRiZ, 12. Jg., 1920, Sp. 106-110, Sp. 107; dicse Auffassung hat sich einige Jahre spärer cine Kölner Gerichtsassessorin zu eigen gemacht. Vgl. Hildegard Koll: Die Frau im Richterberuf, in: Festschrift zur Feier des zjjährigen Bestchens der gymnasialen Sudienansıalı in Köln, 0.0 . 1928, S. 99-104, S. 102 f.

31 Carl: Weibliche Richter?, in: DJZ, 14. Jg, 1909, Sp. 540-\$41, Sp. 541.

32 Diskussionsbeitrag von AGPräs. Riß (Múnchen) während des 4. Richterzags, in: DRiZ, 18. Jg., 1911, Sp. 103

33 Vgl, Eich, a. a. O., Sp. 627.

34 LGDir. Dr. Siadcimane, a. 2. O., Sp. 101.

3) LGRat Dr. Bumke (Danzig), während des 4. Richtertags, in: DRiZ. 18. Jg. 1911, Sp. 20\}.

$36 \mathrm{Vgl}$. Verhandlungen der 14 . Vercrecerversammiung des Deutschen Anwaltsvereins, in: JW, Is. Ig. 1922, S. 1248; die Berufung auf die Verfassung war allerdings zuvor schon auf dem 4. Richtertag verworfen worden: ^... die Reichsverfassung bestimmt, daß grundsätzlich die Frau fur öffentliche Ameer zuzulassen ist. Entscheidend ist das nicht... (DRiZ, 18. Jg., 1921, Sp. 201).

$37 \mathrm{JW} .\{1 . \mathrm{Jg} \ldots 1922, \mathrm{~S} .12\{3$.

$38 \mathrm{Vgl}$. Frgebnis der Vorstandssiczung des Deutschen Richterbundes vom 25.126. 9. 1920, in: DRiZ, i 2. Jg., 1920, Sp. 257-260, Sp. 259; Absimmungsergebnis unter den Teilnehmern des 4. Richecrags, in; DRiZ, $13 . J \mathrm{~g}, 1921, \mathrm{Sp} .206$.

39 I4. Verreterversammlung des Deuschen Anwaltsvercins, in: JW, 51. Jg., 1922, 5. 125$\}.$

40 Allgencine Vertügung vom s. Mai rg19 uber die Zulassung weiblicher Personen zur ersten juristischen Prüfung, in: JMinBl. 1919, S. 288; cine Neuregelung erfolgte am 17.1. 1921 durch die Allgemeine Vcrfügung des preußischen Jusrizmınisters über dic Zulassung zum Vorbereirungsdiensl und zu den juristischen Prïiungen (JMinBI, 192x, S. \{6). - Im Jahre 1921 gabes in PrcuBen is Rechtsreferendarinnen (vg]. Oreo Harrwig, a. a. O., S. is).

41 Vgl Anm. 16 
einzige Konzession bestand darin, daß man den Fraven, soweit sie doch - wie in Baden seit Dezember 1919 - zum juristischen Vorbereitungsdienst zugelassen worden waren, ${ }^{42}$ wenigstens die Vertretungsbefugnis für einen Rechtsanwalt zugestand. ${ }^{43}$ Das Reich stellte sich gegenüber den Forderungen der Frauenbewegung und der Jurastudentinnen auf den Standpunkt, es sei für die Frage der Zulassung der Frauen nicht zuständig; vielmehr müßten die hierzu erforderlichen Schritte von den Landesregierungen unternommen werden. Diese wiederum behaupteten, sie wären an der Zulassung der Frauen zu den juristischen Berufen durch Reichsrecht gehindert. ${ }^{\text {ah }}$

Obwohl endlich 1922 durch Reichstagsbeschluß das "Geserz über die Zulassung der Frauen zu den Ämtern und Berufen der Rechtspflege « eindeurig bestimmte, daß dem weiblichen Geschlecht auch diese Berufe offenstünden, werwuchsen den Frauen doch noch immer Schwierigteiten, die der Mann gleicher Vorbildung nichta hatte. is So verwehre beispielsweise das oldenburgische Justizministerium noch Ende 1929 zwei Assessorinnen die Einstellung in den Justizdienst mit der Begründung, daß es "an hinreichender Erfahrung darüber, ob Frauen sich für jede richterliche Beschäftigung und für alle richterlichen Stellen eignen «, fehle. Es gehe aber nicht an, „Frauen zu Richtern zu machen, die olne jedes Bedenken nur in wenigen Stellen beschäftige werden können ${ }^{46}$ Auch die hamburgische Landesjusrizverwaltung weigerte sich unter ähnlichen Vorwänden, Frauen als Richterinnen zu beschäftigen. ${ }^{47}$ In ganz Deurschland besserte sich die Situation der Juristinnen nach 1922 nur wenig: "Throughour the Weimar years, women were exciuded from the judiciary in Roman Cacholic Bavaria, and although elsewhere in Germany women were eligible for appointment as judges, no woman judge was given a place in the Supreme Court of Justice before the 1950s. Srill in 1930 there was no fernale public prosecuror in relacively liberal Prussia. $\alpha^{48}$

Die Fortdauer der Diskriminierung spiegelte sich auch in der Entwicklung der Zahl der Jurastudentinnen wider: Nach der Aufhebung des Berufsverbors für Juristinnen hatte sich die Zahl der Jurastudencinnen im Wincersemester $1923 / 24$ auf 580 erhöht. Sie sank dann allerdings wieder auf $426 \mathrm{im}$ Wintersemester $192 \$ 25$, steigerte sich im Sommersemester 192S auf 440 und ging bis zum Wintersemester $1925 / 26$ auf 378 zurück. Hatte sich der Anteil der Jurastudentinnen an den weiblichen Siudierenden überhaupt nach der Zulassung der Frauen zu den jurisuischen Berufen im Vergleich zum Sommersemester 1914 merklich erhöht, so spiegelt sich im darauffolgenden Sinken des Prozentsazzes die rasche Desillusionierung wider: Betrug der Anteil im Wintersemester $1923 / 246,2 \%$ und im Wintersemester $1924 / 256,1 \%$, so sank er bis zum Sommersemester 1925 auf $5,8 \%$ und bis zum Wincersemester $1925 / 26$ auf $5,0 \% .19$ Dieser Rückzug der Frauen vom Jurastudium war neben den ungünstigen beruflichen Aussichten für Juristinnen auch auf das bereits an den Universitäten

41 Vgl. Margaretc Berent, 2 1. O., S. 333 .

$43 \mathrm{Vgl}$. Adoll Friedlander, Max Friedlander, a. a. O., S. 76, \$ 25 Anm. 7i zu den Bestimmungen, die nach $19: 8$ in den deutschen Landern im Hinblick sul dic Zulassung der Frauen zur ersien juristischen Seatsprijung und zum Vorbercitungsdienst irgingen, vgl, Vara Lowiesch, ., . . O., S. 63 If.

44 V.l. Margarcte Berent, a. 2. O., S. 334.

4) Käthe Gaebel, a. a. O., S. 218 f.

46 BA Nachiaßs Luders vorl. Nr. i3s.

t7 Vgl. BA Nachiaß Luders vorl. Nr. I3s: Schreiben des Vorsunds der Landesjuscizverwalrung Hamburg vom 3. 12. 1929 an den Deutschen Juriseinnenverein. Vgi. «u dem bamburgiscben und dem oldenburgischen Vorgang außerdem die Schreiben des Reichsinnenministeriums vom 16. 4. 1930 an dic Reichsragsabgeordnere Dr. Marie-Elisabech Lüders und an das Oldenburgische Seatsminiscerium.

48 Jill Stephenson: Wornen in Nazi Society, London 1975, S. 169 l.; noch Ende Juli 1932 gab es in Deurschland keine Nolarin (vgl. Vera Lowitsch، 2. a. O., S. 46 Anm 332).

49 Käıthe Gaebel, 1. a. O. S. 219 . 
herrschende frauenfeindliche Klima - besonders an den juristischen Fakultäten - zurückzuführen. Den Studienbetrieb an der juristischen Fakultät der Universirät Köln beschrieb eine Studentin folgendermaßen: „Eine Studentin braucht nur den Mund aufzutun, ganz gleich, ob zu guter oder zu schlechter Antwort, und es wird nach deutschakademischer Sitte getrampelt, gescharrt oder blöd gelacht. Ist im Kolleg die Rede von den vorsintflutlichen Bescimmungen unseres Familienrechts und von vergangenen herrlichen Zeiten, als die Frau noch ganz unter ehelicher Gewalt stand, zeigen die Jünger am Geiste auf ebenso böfliche Weise begeisterte Zustimmung. Moderne Reformbestrebungen mißbilligen sie aufs schärfste. Als einmal ein Professor sagte, die Frauen könnten jetzr auch Handelsrichterinnen werden, war das ganze Kolleg so tief entrüstet, daß der Dozent erst nach zehn Minuten fortfahren konnte. Kommt eine Srudentin zu spät ins Kolleg-die Herren erlauben sich das häufiger -, wird hefrig gescharrt. «"

Der Anteil der Frauen an der Gesamtheit der Jurastudenten betrug im Wintersemester $1925 / 26$ etwa 2,5\%. ${ }^{\prime \prime}$ Noch stärker uneerrepräsentiert waren die Frauen in den juristischen Berufen: Bei der Berufszählung vom I6. Juni i 92 s wurden 55 Rechrsanwältrinnen ermitrelt. An der Gesamtheit der Rechrsanwälte stellıen die Frauen damir einen Anteil von nur $0,4 \%$.' Auch acht Jahre später hatte sich ihr Anteil nicht nennenswert erhöht: Der Berufszählung vom I6. Juni 1933 und der saarländischen Ergänzungszählung vom 25. Juni 1935 zufolge betrug der Frauenanteil an den insgesamt 18766 Rechtsanwälten und Notaren mit 252 Personen r, $3 \%$." Der Anteil der Frauen an den Richtern und Sraatsanwälten belief sich :933 (Saarland; 1935) auf $0,3 \%: 10405$ Männern standen nur 36 Frauen gegenüber. ${ }^{94}$

Mit der faschiscischen "Machtergreifung" setzte nicht nur für die Akademikerinnen allgemein, sondern gerade auch für die Juristinnen eine Periode zunehmender Diskriminierung ein. Daß die NSDAP der Juristin feindselig gegenüberstand, batte bereirs 1931 Joseph Goebbels im "Angriff" unmißverständlich dargelegt: "So ist neuerdings die Assessorin Spitzer Vorsitzende des Schöffengerichts Charlottenburg geworden. Wenn dann vielleicht noch, wie in der ersten Sitzung, noch ein weiblicher Schöffe vorhanden isc, das Gerichr also mit zwei Frauen und einem Manne besetzı ist, so bedeutet das eine Herabwürdigung der Rechtspflege, wie sie ärger kaum gedacht werden kann. «" Die ersien Ansätze zur Ausschließung der Frauen von den juristischen Berufen wurden durch das «Geserz über die Zulassung zur Rechtsanwalrschaft» vom 7. April $1933,{ }^{66}$ die neue Jusrizausbildungsordnung (JAO) vom 22. Juli $1934^{\text {s7 }}$ und die Aulhebung der Rechtsanwaltsordnung vom 1. Juli $1878^{88}$ durch die nationalsozialistische Reichsrechtsanwalisordnung vom 21. Februar $1936^{19}$ anternommen. Wenn auch keine dieser Bescimmungen das Verbor

50 Abendblatt der Frankfunter Zeirung vom 6. 1. 1930, zil. n. Michael H. Karer: Krisis des Frauensrudiums in der Weimarer Republik, in: VSWG 59,1972, S. 207-2 is, S. 124 f.

st Karhe Gaebel, a.a.O. S. 220 ; dahingegen stelle sich der Fraucnanteil an den Sudierenden der philologisch-hiscorischen Wissenschaften auf $26 \%$, der Pharmazic auf $20 \%$, der Machemarik und Naturwissenschaften uf $17,75 \%$, der Medizin auf $16,3 \%$ Bei den Volkswirischafisstudenten betrug der Frauenantcil $9,3 \%$, bei den Chemiestudenten $6,8 \%$ und selbst bei den Studierenden der crangelischen Theologic noch $2,8 \%$.

51 SUDR Bd. 408, Berlin 1931, S. 299. Dic ersıen Anwâltinnen hatıen sich im Jahre r 924 niedergelassen (vgl. Maric Munk: Die Juristin. Berlin 1918, 5. 7).

13 SIDR Bd. 470, 2, Berlin 1937, S. 47.

s4 A. 2. O., S. 46 ; zum Vergleich der Frauenanteil in anderen Berulen: Ärze 8,5\%, Zahnärzte $10.3 \%$, Aporheker 20,4\%, Volks-, Mirrel., Ober-, Beruss- und Fachschullehrer 30,1\% (vgl. a. a. O., S. 47 I.).

is Zit. n. Gewerkschafiliche Frauenzeinung, is. Jg. Nr. 4, Berlin, is. 4- $2931,5.30$.

\$6 RGBI. 1933 I, S. 188 .

37 RGBI. 1934 1, S. $727-736$.

s\& RGBI. $1878,5.177-198$.

59 RGBl. 1936 I, S. 107-118. 
weiblicher Beteiligung an den juristischen Berufen ausdrücklich aussprach (wodurch eine gewisse Verschleierung der tarsächlichen Absichten erreicht wurde), so fiel doch einigen juriscisch geschulten Personen auf, welche Möglichkeiten der Ausschließung sich aus den neuen Vorschriften ergaben. So erkannte Max Wagner in seinen 1934 erschienenen Kommentar zur $3 \mathrm{AO}$, daß diese eine Reihe von Bestimmungen enthieir, "aus denen sich zwingend die Schlußfolgerung ergibc, daß nach dem Willen des neuen Gesetzes nur Männer zur ersten juriscischen Staarsprüfung zugelassen werden dürfen. « ${ }^{60}$ Dagegen vertraten der Präsident des Reichs-Justizprüfungsamts, Otto Palande, und der Ministerialrat im Reichsjustizministerium (RJM), Heinrich Richrer, in ihrem Kommentar zur JAO die Ansiche, daß, da das Gesetz über die Zulassung der Frauen zu den Ämtern und Berufen der Rechtspflege vom I 1. Juli 1922 noch in Kraft sci, den weiblichen Jurastudenten die Zulassung zur ersten juristischen Staatsprüfung und zum Rechtspraktikum auch durch die neue JAO nicht verwehrt werde. ${ }^{6 r}$ Palandt und Richter betonten allerdings, "daß es Sache des Mannes ist, das Recht zu waluren ${ }^{6}{ }^{62}$ womit sie sich eindeutig von dem in der Weimarer Reichsverfassung niedergelegten Gedanken der Gleichberechtigung der Frau distanzierten.

Bereits zu dieser Zeit konnten Juristinnen nach bestandener zweiter Staatsprüfung nicht mehr darauf hoffen, als Assessorinnen in den preußischen Staatsdienst übernommen zu werden, ${ }^{6}{ }_{3}$ und in den anderen Ländern dürfte die Lage ähnlich gewesen sein. Dies entsprach im übrigen vollkommen dem Programm „zur unbedingt notwendigen Entpólicisierung der Frau und ihrer Arbeir ", das Paula Siber, später stellvertretende Leitexin des "Deutschen Frauenwerks«, bereits im August 1933 als Beauftragte des Referats für Frauenfragen beim Reichsinnenminister (RMdI) vorgelegr harte, und in dem es u. a. hieß: „Das eigenwerrig frauliche Arbeitsgebiet führt von der Familie als dem Fundament von Volk und Staat zur großen Volksgemeinschaft hin. $x^{6 ;}$

r935 wurde schließlich die künfrige Schließung des öffentlichen Dienstes für Frauen von seiten des RJM vorgeschrieben: „Der Herr Reichsminister der Jussiz beabsichigt nicht, Frauen in die Richter- und Staatsanwaltslaufbahn zu übernehmen. Wer bis zum 31.3 .1936 bereirs in das Beamtenverhältnis übernommen ist, bleibr in seiner Stellung. Alle anderen Aufträge werden bis spätestens zum 31. 3. 1936 widerrufen werden. $\ll^{63}$

Proceste der Frauenbewegung oder der betroffenen Juristinnen waren zu diesem Zeitpunkt nicht mehr zu erwarten: War jene bereits 1933 zerschlagen worden und konnte daher auch nicht mehr ihre Stimme erheben, so waren auch diese noch 1935 im unklaren über ihre Situation gelassen worden, so daß keine ernstzunehmende Bewegung gegen den durch die Hintertür sich hereinschleichenden Juristinnenabbau zustandekommen konnte. In welch geringem Maße wahrscheinlich sogar die "Reichsfrauenführungu, deren Haltung zur rechtlichen Stellung der akademisch

60 Max Wagner: Justizausbildungsordnung des Neuen Reiches mit Eriaureruogen, Köln o. J. (1934), S. 3s.

61 Ono Palandu, Heinrich Richter: Die Justizausbildungsordnung des Reiches, 2. crw. Aufl. Berlin r939. S. 6: die Zulassung zu den Staasporüungen wurde den Frauen tassächlich niche verweigert, vpl. Die akademischen Berufe. Die Recheswahrerin (Jurisuin), brsg. v. Akademischen Auskunfusame Berlin, 2. Auff. Berlin 1941, S. 12.

6. Ono Paland, Heinnch Richter, 2. a. O., S. 6.

6) Vgl. Walier A. Boje: Frauen in Zukunfi nicht mehr zu den juristischen und ierärztlichen Prüfungen zugelassen?, in: Die Frau, 41. Jg, 1934/35, S. 231-234, S. 2\}2.

$64 \mathrm{BA} \mathrm{R}_{43}$ [I/82; a: Schreiben des Referats für Frauenfragen beim RMdI (Paula Siber) vom 13. 8. 1933 an Staatssekretär Dr. Hans-Heinrich Lammers in der Reichskanzlei.

6s Erlaß des RJM vom 17. 9. 193 s an die Oberlandesgerichispräsidenten und Generalstaatsanwäke, zit. n. Anne-Gudrun Meier-Scherling, a. a. O., S. 11; dieser Erlaß whrde in seinen wesentlichen Ausfuhrungen durch cin Rundschreiben vom 20. 1. 1936 wiederhole (vgl. cbenda). 
gebildeten Frau durchaus niche in jedem Einzelfall mit derjenigen der Parteiführung übereinstimmte, ${ }^{66}$ über die beginnende Diskriminierung der Juristinnen informier war, ${ }^{67}$ beleuchtet dex folgende Vorgang: Als 1937 durch das RMdI auf Anfrage des Amrs des "Stellvertreters des Führers" ein Erlaß an die obersten Reichsbehörden herausging, in dem (estgestellt wurde, daß Hiler ogrundsärzlich nur die Ernennung von Männem zu Beamten des höheren Dienstesw wünschte, bat das RMdI zugleich in einem Schreiben an die Reichskanzlei wum Mitreilung, ob Bedenken dagegen bestehen, den vertraulichen Erlaß ... der Reichsfrauenführung mitzuteilen. ${ }^{68}$ Offensichtlich war es also durchaus angängig, die "Reichsfrauenführung ü̈ber Vorgänge, die sie direki betrafen bzw. in die Rechte der von ihr angeblich vertretenen Bevölkerungsgruppe eingriffen, nicht in jedem Falle in Kennenis zu setzen. Diese Zurückhalrung in der Informauionspolitik gegenüber der »Reichsfrauenführung* nährt die Annahme, daß das Vorgehen gegen die Juriscinnen zumindest bis zu jenem Zeitpunkt, da ihnen die Berufsausübung endgültig und umfassend (d. h. alle juristischen Berufe betreffend) verwehrt war, selbst der faschistischen Frauenorganisation nicht bekannt war, so daß auch diese an einem eventuellen Protest gehindert war. Das erste Dokument, in dem das künfrige Berufsverbor für weibliche Juristen durch Hitler selbst bestätigt wurde, stammt vom 24. August 1936. In einem unter diesem Datum abgefaßten Schreiben an den RJM reilte Martin Bormann im Auftrag von Rudolf Heß mit: nAuf Grund der Besprechung in Ihrem Ministerium vom s. August 1936 wegen der Zulassung von Fraven zur Anwaltschaft habe ich, da, wie der Verlauf der Sirzung zeigce, die Parrei an diesen Dingen auch ein besonderes Interesse hatte, dem Führer die Angelegenheir vorgerragen. Er hat entschieden, daß Frauen weder Richter noch Anwalt werden sollen. Juristinnen können deshalb im Staatsdienst nur in der Verwaltung verwandt werden. Wegen der vorhandenen Assessorinnen bitre ich bemüht zu sein, diese nach Möglichkeit dort unterzubringen. ${ }^{69}$ Durch diese Entscheidung wurden nun nicht mehr nur diejenigen Juristinnen, die Richterin oder Scaassanvältin werden wollten, an der Ausübung ihres Berufes gehindert, sondern auch die angehenden Rechtsanwälcinnen. Der Bruch der Weimarer Verfassung war also auch in dieser Hinsicht vollzogen worden, und obwohl das Gesetz über die Zulassung der Frauen zu den Ámtern und Berufen der Rechisptlege von 1922 de iure noch immer in Kraft war, hatte es de facto keine Relevanz mehr.

Der Abbau der bereits auf Widerrul oder auf Lebenszeit verbeamteten Juristinnen bereitete kaum Schwierigkeiten: Von den wenigen 1933 im öffentlichen Dienst tätigen Juristinnen waren einige bereits im Zuge der Säuberung des Beamtenapparares enclassen worden; die wenigen, die übrig geblieben waren, wurden nach und nach durch Versetzung auf Verwaltungsstellen abgeschoben ${ }^{70}$ und waren *von jeder Beförderung ausgeschlossen a. "Ahnlich erging es auch den freiberuflich tätigen Rechtsanwältinnen: 1933 war einem Teil von ibnen aus politischen Gründen

66 Vgl. Jill Srephenson, a. a. O., S. 173 f.

67 Dic Fernhaleung der sReichsfrauenführung* selbst van solchen Enrscheidungen, die die weibliche Bevölkerung unminclbar betrafen, entsprach cinem BeschluB aus der Frühzeit der NSDAP. Vgl. Georg Franz-Willing: Die Hirlerbewagung, Bd. I, Hamburg/Berlin 1962, S. 82.

$68 \mathrm{~V}$ l. BA R 43 [1/427: Schreiben des SLabs des Stelliventreters des Führers vom 4. 5. 1937 an die Reichskanzlei. Schreiben des RMdI rom 17. 11. 1937 an den Chef der Rciehskanzlei. Die Reichskanalei beailligie übrigens mit Antwortschreiben vom j1. 1. $193^{8}$ die Lnterricheung der *Reichsfrauenlühring $\alpha$.

69 BA R $43[1 / 417$.

70 BA R 4] II/427: Schreiben Dr. Freislers im RJM vom 16. 1. 1937 an die obersten Reichs- und preußischen Behörden.

7 Annc-Gudrun Meier-Scherling, 2. 2. O., S. 10. 
Berufsverbor erreilt worden, ${ }^{72}$ seit Ende 1935 wurden Fraven nicht mehr zur Anwaltschaft zugelassen und am 31 . März 1936 endeten alle Kommissorien für síe. ${ }^{73}$ Etwas günstiger sah es zunächst für die abhängig beschärtigten Anwältinnen aus: Sie konnten weiterhin in einer Anwaltspraxis arbeiten. Allerdings war auch ihnen die Vertretung ihrer Mandanten vor Gericht untersagt, so daß längeríristig ein seibständiger Anwalt kaum Inreresse an einer nur bedingr einsarzfähigen Arbeitskraft haben konnte und daher im Laufe der Zeit wohl die meisren abhängig beschäfrigten Rechtsanwältinnen ihren Arbeitsplarz verloren.

Angesichrs dieser Sachlage kann es nur als Schimäre bezeichner werden, wenn Erwin Noack in seinem 1937 erschienenen juristischen Kommentar zur Reichsrechtsanwaltsordnung schrieb: „Frauen sind hinsichelich der Zulassung zur Rechtsanwaltschaft den Männern völlig gleichgestellt. (74 $^{74}$

Infolge der Berufsverbote für Juristinnen sank die Zahl der Jurastudentinnen in Deutschland. Hatte sie 1932, als der Anteil des weiblichen Geschlechts an den Studierenden seinen höchsten Stand vor dem Zweiten Weltkrieg erreichte, "7 noch über tausend betragen, so wurden im Wintersemester 1937/38 nur noch $59 \mathrm{im}$ Fach Rechtswissenschafren immatrikulierce Frauen gezählt, ${ }^{6}$ weniger also als im Sommersemester 1917, als i 17 Frauen Jura studierren. ${ }^{77}$

Als mir der Entfesselung des Zweiten Weltkriegs durch die Einberufungen männlicher und das Berufsverbor weiblicher Juristen die Knappheir an "Rechrswahrern * offensichclich wurde, ${ }^{78}$ uncernahm der $n$ NS-Rechtswahrerbund einen Vorstoß in Richtung auf eine planmäßige Anstellung von Referendarinnen und Assessorinnen bei den Stadtverwaltungen, nachdem man festgestellt hacte, daß sowohl eine Betärigung auf dem Gebiece der sozialen Arbeit ${ }^{79}$ als auch eine Beschältigung in ngehobene(n) Sekretärinnenstellungen « "beinahe ausnahmslos von den weiblichen Rechrswahrern abgelehnt " wurden. ${ }^{80}$ Solche und andere Versuche, die Frauen zumindest während des Zweiten Weitkriegs in beschränktem Maße wieder juristisch (wenn auch nicht in den eigentlichen juristischen Berufen) arbeiten zu lassen, ${ }^{8}$ stießen jedoch zunächst auf Widerstand. So betonte der Generalbevollmächtigte für die Reichsverwaltung (GBV) im Jahre 1940 nim Einvernehmen mit dem Stellverreter des Führersa, daß auch weiterhin wnach einer Entscheidung des Führers die Anstellung von Frauen als Richterin oder ihre Zulassung als Rechtsanwältin aus(scheide). ${ }^{82}$ Erst 1942 wurde diese grundsätzliche Beschränkung der Berufsausübung durch Juriscinnen im öffentlichen Dienst etwas gelockert. Am 16. Januar schrieb der Stazessekretär im RJM, Roland Freisler, an die Oberlandesgerichrspräsidenten: „Die Einberufungen zur Wehrmache haben zur Prüfung der Frage geführt, ob unter den obwaltenden Verhälenissen ausnahmsweise auch Frauen mit der

72 Das prominenteste Beispiel hicrfur durlte wohl die Berliner Rechtsanwältin und spätere juscizministerin der DDR, Hulde Benjamin, sein (vgl. Jill Siephenson, a. 3. O., S. 1,6).

73 Vgl. Anne-Gudrun Meier-Scherling a. a. O., S. ro.

74 Erwin Noack: Kommentar zur Reichsrechtsanwalısordnung in der Fassung vom 21. Februar 1936, 2. neubearb. Aufl. Leipzig 1937 , S. 28.

75 Im Wineersemester r931/32 belief sich der Frauenanteil an der Scudentenschafe in Deurschland aul $18,9 \%$ (vgi. Jill Stcphenson, a. a. O., S. I31).

76 Vgl. a. a. O., S. 138

77 Vgl. Hilde Oppenheimer, Hulde Radomski, a. 2. O.. S. 157.

$78 \mathrm{Vgt}$. Jill Stephenson, a. a. O., S. 139.

79 Hierzu zählten die Jugendpllege und fürsorge, die Volkspilege, die Arbeit bei der weiblichen Kriminalpolizei und auch die Tärigkeit als Juristin beim Reichsarbeirsdienst für die weibliche Jugend.

80 Vgl. Deutsches Rechi. Zentralongan des NS-Rechtswahrerbundes, Ausgabe A, 9. Jg., 1939, 5. 1629.

8. Vgl. Margarete Esch: Lage und Aussichten in den höheren Fraucnberulen, in: Dic Frau, ${ }^{9}$. Jg.. $19,0 / 41$, S. $168-173$, S. 168 .

82 BA R ${ }_{43}$ II/427: Schreiben des GBV vom 20. 5, 1940 an die oberscen Reichsbehörden. 
Befähigung zum Richteramt nach $\$ 7$ LaufbVO verwender werden dürften. Auch im Kriege muß der Grundsatz aufrechterhalten bleiben, daß eine Frau weder als Richter (Staatsanwalt) noch als Rechtsanwalt tätig werden soll. Immerhin halce ich es angesichts der schwierigen Personallage für vertretbar, $d a ß$ zur Behebung eines Notstandes eine Frau ausnahmsweise bei größeren Gerichten zur Bearbeitung von Grundbuch- oder Registriersachen - also in einer mehr verwaltenden Tätigkeit - eingesetzt wird. Dagegen ist die Verwendung als Vormundschafts-, Nachlaß-und Konkursrichter und selbstverständlich als Prozeßrichter nicht angängig. Ich darf anheimstellen, entsprechend zu verfahren. c $^{83}$ Diese noch recht rigorose Verfügung wurde schon zwei Monate später dahingehend abgeschwächt, daß nun Juristinnen vauf allen Gebieten der freiwilligen Gerichtsbarkeit verwendet werden k konnten, walso in weiterem Umfange a's durch die RV vom 16. 1. 1942 zugelassen worden ist. Im übrigen bleiben die Grundsätze der RV in Gülcigkeit (Verwendung nur zu Behebung eines Personal-Notstandes für die Kriegsdauer, Verwendung regelmäßig nur bei größeren Gerichten). $\alpha^{84}$

Als Anfang 1944 das "Amt für Neuordnung der Deurschen Gerichtsverfassung* beim RJM anfragte, wie vieje Frauen noch Planstellen als Richterinnen innehäcten, konnte diese Frage niche mehr beantwortet werden, »da die Kartei verbrannt isi $\alpha$. Das RJM versichere aber, dem einen bekanntgewordenen Fall einer Einzelrichrerin in Ehesachen an einem Mannheimer Gericht unverzüglich nachgehen und nötigenfalls diese Frau aus ihrem Amt entfernen zu wollen. ${ }^{\text {ss }}$ Sollte diese Mannheimer Richterin tatsächlich die einzige im Jahre 1944 im Deutschen Reich täcige Richterin gewesen sein, so kann daraus auf die Fortführung des Richterinnenabbaus auch während des Zrveiten Weltkriegs geschlossen werden, denn noch am 1. Juni 1939 waren neun Frauen planmäßig als Richterinnen beschäfrigr, davon fünf außerhalb Preußens. ${ }^{96}$

Noch intransigenter als mir den Juristinnen des öffentlichen Dienstes wurde mit den Rechrsanwältinnen verfahren: Ihnen gegenüber fand keine Lockerung der apodiktischen Diskriminierung statt. "Zwar konnce eine Juristin als amclich bestellie Verreterin eines Rechtsanwalts fungieren. Einige Juristinnen haben wäbrend des Krieges die Praxis eines eingezogenen Kollegen geführt. Wenn der Vertretene fiel, endete auch ihre Vertrerung; sie durften die Praxis nicht fortführen. Das war besonders hart, wenn der Gefallene der Ehemann der Vertreterin gewesen war. ${ }^{87}$ Als sogar im RJM zur Uberbrückung der Personalknappheit während des Zweiten Welkkriegs der Gedanke an eine zumindest für solche Wirwen zu gestattende Führung einer Rechtsanwaltspraxis aufkam und am 16. Mai 1944 ein entsprechendes Schreiben an die Partei-Kanzlei gesandt wurde, kam von dort ein abschlägiger Bescheid: „Der Führer entschied bereits im Jahre 1942 zu der von Ihnen mit Schreiben vom i6. 5 .

83 BA R 22/232.

84 BA R 22/232: Schreiben des Staztssekretïrs im RJM, Dr. Frcisler, vom 27. 3. 1942 an die Oberlandesge. richesprasidenecn, Gencralstaatsanwälte und an Marrin Bormann.

8) Vgl. BA R 22/2\}2: Antwortschreiben der $\Lambda$ bt. 1 im RJM an Ministerialdirektor Alistocter im Amt für Neuordnung der Deuschen Gerichisveriassung vam 8. 2. 1944 auf dessen Anirage vom 20.1. 1944. Der für Mannhcim zusıändige Obcrlandesgerichespräsident in Karlsnuhe wurde am 4. 7. 1944 vom R ]M darauf aufmerksam gemachu, daß ndic Anardnungen über die Verwendung von Frauen im richerlichen Dienst io der RV vom 16 . Januar 1942 nicht geandert worden (sind). Wenn diese RV daraú hinweist, daß die neu eingestellien Frauen nur aul bestimmen Gebieren der Jreiwilligen Gerichtsbarkeil verwendec werden dürien, so hat sie hiermi nicht eine allein auf die neu... eingestellen Fraven beschränke Anordrung usflen wollen. Die RV gehe vielmehr, woran ihre Fassung keinen Zweilel läßr, davon aus, daß alle im richeeplichen Dienst verwenderen Frauen ausschließlich aú dem Gebiete der frciwilligen Gerichesbarkeit Latig sein durlen ....

$86 \mathrm{Vg}$. BA R 22/2\}2: Schreiben der Abı. I im RJM vom 31. 3. 1944.

8y Anne-Gudrun Meicr-Scherling, 2. a. O., S. 12. 
1944 angeschnittenen Frage, er wünsche nicht, daß Assessorinnen, deren Ehemänner gefallen sind, zur Rechisanwalischaft zugelassen werden, da derartige Maßnahmen sich auch nach dem Kriege auswirken würden... Im Hinblick auf die eindeutige Weisung des Führers möchte ich davon absehen, ihn erneur zu belragen. ${ }^{88}$ Diese seindeutige Weisung a Hitlers machte die Unwahrheit seiner Darstellung der beruflichen Rechre und Chancen der Frauen im «Dritten Reich sichtlich. In einem Interview hatte er 1936 dazu ausgeführt: "Ich gebe den Frauen das gleiche Recht wie den Männern, aber ich glaube nichr, daß sie îhnen ähnlich sind ... Aber in jedem Fall hat eine Frau, die niche heiratet (!) . .. das Recht, ihren Lebensunterhalı zu verdienen wie der Mann.a ${ }^{89}$

Die Lage der Juristinnen war also durch eine anhaltende, mal mehr, mal weniger scharfe Diskriminierung geprägt. Auf keinem anderen Gebiet (abgesehen von dem der römisch-katholischen Theologie; hier spielen aber andere als in der deutschen Politik begründete Fabcaren die entscheidende Rolle) hat die Akademikerin in Deutschland eine solche Fülle von Herabseczungen und Behinderungen erfahren müssen. In der Haltung gegenüber den Juristinnen bündelten sich nicht nur all die vielfältigen Formen der Diskriminierung weiblicher Akademiker, sondern es traten zusärzliche hinzu, von denen das Berufsverbot die earscbeidendste war. Zwar wurde den Juristinnen während rund zwölf der ersten 4s Jahre dieses Jahrhunderts eine ihrer Ausbildung gemäße Betätigung offiziell gestartet, doch bestand auch in dieser Periode die uneingeschränkte Gleichstellung mit den männlichen Kollegen lediglich auf dem Papier, aut das das Gesetz über die Zulassung von Frauen zu den Ämtern und Berufen der Rechtspflege gedruckt war. Unter Berufung auf die angeblich win den Tiefen der Seelea zu suchenden wseelischen Geschlechrsunterschiede ${ }^{90}$ wurde über Jahrzehnte die Frau in Unmündigkeit und Unselbständigkeit gehalten, und wo dies nicht half, offener Repression ausgesetzt. Am Ende dieses Weges stand die terroristische Diktatur, die, in der Kontinuität der Frauenfeindlichkeit der konservariven Rechten und der Kirchen stehend, die Frauen wieder verstärkt unter die patriarchalisch-autoritäre Familienform zwang und ein auf dem Männlichkeitswahn des "deutschen Herrenmenschen* gegründetes inhumanes Herrschafrssystem errichtete.

88 Marcin Bormann am I1. 8. 1944 an das RjM, zir. n. Anne-Gudrun Meier-Scherling, a. 2. O., S. 12. 89 Hitler am 23. 1. 1936 in einem Interview mit der französischen Joumalistin Titayna, in: Max Domarus: Hitler. Reden und Prokizmationen 1932-1945, Bd. 1, Würzburg 1962, S. $\{6 \%$

90 Martha Moers: Dic Seclenlage der Frau im Berricb, in: Arbeir und Berrieb, 14. Jg., 1944, S. 13-19, S. 14. 


\section{Rolf Bender/Rolf Schumacher \\ Erfolgsbarrieren vor Gericht}

Eine empirische Untersuchung zur Chancengleichheit im Zivilprozeß. 1979. XI1, 150 Seiten. Kart. DM 29.-

Die Rechtssoziologie - nicht nur in der Bundesrepublik - befaBt sich seil langem mit dem Problem der »Erfolgsbarrieren vor Gericht", die oftmals als »Klassenjustız tituljert werden. Bislang liegen darüber fast nur (heorerische Spckulatiouen, Einzelfallanalysen oder berufssoziologische Untersuchungen übcr Herkunft und Einstellung von Richtern vor.

Wir wissen zwa r inzwischen, wievicle Richler einen katholischen Vaicr haben, der vom Lande stammi und millerer Beamter war; darüber aber, ob und wie sich diese Umstände auf die Entscheidungsfindung auswirkt, gibt es nur Spekulationen.

Hier wird erstmals auf einer wirklich breiten empirischen Grundlage (Analyse von ca. 8000 Zivilprozeßakten) versucht, Klarheit darüber zu gewinnen, ob es eine schichtspezifische Benachteiligung vor Gericht gibt, welches $A u s m a B$ sic hat und worauf sic beruht.

Gleichzeitig wird anhand dieses recluspolitisch brisanten Themas der Gang und die Auswertung einer derartigen sozialwissenschafilichen Analyse - für jedermann nachvollzichbar dargestellt. Dies dürfte vor allem für die Juristen inceressanı sein, die die Soziologie bislang häufig nur von den Meinungsum. fragen und sog. "Juslizbeschimpfungen " her zu kennen glauben. von der wirklichen Leislungsfähigkeit der Soziolgie aber wenig Ahnung haben.

Ferner haben die Verfasser das umfangreiche Material auch auf viele andere Fragestellungen hin aufbereitel. Dabei dürftcn insbesondere die Ergebnisse der empirischen Überprüfung der von vielen Justizreformern behaupteten "Qualitätsunterschiede" der Rechtsprechung der kleinen und großen Gerichte eine echte Überraschung darstcllen.

Am SchluB werden Folgerungen diskutierl, die sich aus den Ergebnissen der Untersuchung für die Gesetzgebung aufdrängen.

\section{J.C.B.Mohr (Paul Siebeck) Tübingen}

